\title{
Experimental Characterization of Aerosol Suspension in a Rotating Drum
}

\author{
Sheng-Hsiu Huang ${ }^{1}$, Yu-Mei Kuo ${ }^{2 *}$, Chih-Wei Lin ${ }^{1}$, Wei-Ren $\mathrm{Ke}^{1}$, Chih-Chieh Chen ${ }^{1 * *}$ \\ ${ }^{I}$ Institute of Occupational Medicine and Industrial Hygiene, College of Public Health, National Taiwan University, Taipei \\ 10055, Taiwan \\ ${ }^{2}$ Department of Occupational Safety and Health, Chung Hwa University of Medical Technology, Tainan 71703, Taiwan
}

\begin{abstract}
Rotating drum chambers are simple and effective devices for retaining particles in the airborne state for prolonged periods. Many studies, including inhalation toxicology, environmental fate, and survivability of airborne pathogens, can benefit from using them. Particle size is the major factor governing aerosol suspension, yet reliable experimental data on the optimal rotation rate as a function of particle size are limited. Therefore, this study aims to experimentally characterize a rotating drum and to optimize the rotation rate for the bioaerosol size spectrum. Moreover, the sampling methodology for evaluating the performance of a rotating drum is investigated. Charge-neutralized potassium sodium tartrate (PST) particles generated by an ultrasonic atomizer are used as surrogates of bioaerosols to characterize the performance of the rotating drum. The aerosol number concentrations and size distributions in the rotating drum are both continuously and intermittently measured by an aerodynamic particle sizer (APS) in real time. Then, the decay constants of the aerosol number concentrations as functions of particle size, elapsed time, and rotation rate are calculated. The experimental results reveal that the rotation of the drum chamber greatly enhances particle suspension and the rotation rate can be optimized to prolong the suspension for an extended period. With the current drum geometry, the optimal rotation rate varies from 2 to $7 \mathrm{rpm}$ for the particle size range of 1 to $7 \mu \mathrm{m}$ and is proportional to the particle size. At the optimal rotation rate (2-4 rpm) for $1-\mu \mathrm{m}$ particles, $5 \%$ of them can remain suspended for over 24 hours. However, this rate must be adjusted for large particles in order to maximize the period of suspension.
\end{abstract}

Keywords: Rotating drum; Decay constant; Suspension time; Optimal rotation rate.

\section{INTRODUCTION}

Many infectious organisms including viruses, bacteria, and fungi have been shown to be transmitted by the airborne route. The ability of these microorganisms to survive in the airborne state plays a key role in their aerial dissemination. Therefore, besides recommending good personal hygiene practices, many researchers tried to formulate environmental control guidelines through investigating the effects of factors such as temperature, relative humidity, and light or chemicals exposure on promoting or retarding the survival of infectious organisms in the air. However, results on airborne survival of microorganisms from previous studies varied considerably due to different experimental techniques

\footnotetext{
* Corresponding author.

Tel.: 886-912017249; Fax: 886-6-2894028

E-mail address: ymkuo@mail.hwai.edu.tw

** Corresponding author.

Tel.: 886-928253663; Fax: 886-2-23938631

E-mail address: ccchen@ntu.edu.tw
}

used. Thus, a well-characterized experimental system is essential for making generalized conclusions and developing mathematical models.

Many measures have been developed to control the infectiveness of airborne pathogens (Huang et al., 2012; Chen et al., 2016). The key to validate the effectiveness of control measures is to retain the microorganisms in aerosol phase for prolonged periods of time. However, the suspension of bioaerosols is subjected to gravitational settling in a stationary test chamber. Although keeping the aerosols in stationary chambers with gentle stirring would delay sedimentation, large particles still need long settling distances to extend the airborne period. Alternatively, a rotating drum (Goldberg et al., 1958), which is an uncomplicated but more effective device, was shown to be capable of retaining particles in airborne state for prolonged periods. Experimentally, as compared with a stirred settling aerosol chamber $1 \mathrm{~m}$ in height, almost a 20 -fold increase in duration of holding time for $1-\mu \mathrm{m}$ particles in a $1-\mathrm{m}$-diameter drum chamber rotated at $2 \mathrm{rpm}$ is demonstrated (Goldberg, 1971). Another subsequent study generated di-n-hexyl phthalate particles with a $0.8-\mu \mathrm{m}$ mass median diameter in a $40-\mathrm{cm}-$ diameter drum and found a $90 \%$ reduction of the particle 
settling loss as the drum rotated at about $3 \mathrm{rpm}$ (Frostling, 1973). However, bacterial and fungal aerosols in indoor environment are usually in the size range of $3 \mu \mathrm{m}$ in count median diameter (CMD) which is much larger than those tested in the previous studies (Kuo, 2015). Even though the aerosol decay as a function of particle size in a rotating drum chamber has been comprehensively studied using advanced particle sizing instruments (Embury and Sutton, 2005; Sutton, 2005), several issues that can affect the results have not been addressed. Since aerosol charge neutralizers were not used, their data would reflect the influence of particle charge. Furthermore, the high particle number concentrations used in these studies might cause significant coincidence errors in the APS counts.

In rotating drum chambers, particles remain suspended as a result of the competition between gravitational and centrifugal forces. At high drum rotation rate, the centrifugal force is the dominant aerosol deposition mechanism. Taking into account the combined effects of gravitational and centrifugal forces, Gruel et al. (1987) developed an equation to predict the fraction of suspended particles by assuming that the aerosols in the rotating chamber decay exponentially (referred to as Gruel's model). The fraction of suspended particles is:

$$
\frac{N}{N_{0}}=\left[\frac{\left(R-r_{0}\right)^{2}}{R^{2}}\right] \exp \left(-2 \tau \omega^{2} t\right)
$$

where $N$ and $N_{0}$ are number of particles and initial number of particles in drum, respectively; $R$ is the drum radius; $r_{0}$ is the radius of gyration of a particle in the rotating drum $\left(r_{0}=\tau g / \omega\right) ; \tau, g$, and $\omega$ are the particle relaxation time, gravitational acceleration, and angular velocity of drum rotation, respectively; and $t$ is the time beginning after one revolution.

Gruel's model is appropriate for predicting the fraction of suspended particles after one revolution when centrifugal force is much less than gravitational force (Embury and Sutton, 2005). However, it is not applicable when the rotation rate is reduced to zero because the solution does not converge to the gravitational settling solution (Asgharian and Moss, 1992). Therefore, Asgharian and Moss (1992) used the concept of limiting trajectories of the suspended particles that was proposed by Pich (1972) to predict the fraction of suspended particles as a result of the combined effects of gravity and centrifugation over the complete range of rotation rates (referred to as Moss's model). The fraction of suspended particles is:

$$
\begin{aligned}
& \frac{N}{N_{0}}=1-\frac{1}{\pi}\left[\frac{y_{p}}{R} \sqrt{1-\left(\frac{y_{p}}{2 R}\right)^{2}}+2 \sin ^{-1} \frac{y_{p}}{2 R}\right] \text { for } 0 \mathrm{rpm} \\
& \frac{N}{N_{0}}=\exp \left(-2 \tau \omega^{2} t\right) \text { for } \frac{H}{R} \leq 1-\exp \left(-\tau \omega^{2} t\right)
\end{aligned}
$$

$$
\begin{aligned}
\frac{N}{N_{0}}= & \frac{1+\exp \left(-2 \tau \omega^{2} t\right)}{2}-\frac{1}{\pi}\left[\frac{H_{1}}{R} \sqrt{1-\left(\frac{H_{1}}{R}\right)^{2}}+\sin ^{-1} \frac{H_{1}}{R}+\right. \\
& \left.\frac{H_{2}}{R} \sqrt{\exp \left(-2 \tau \omega^{2} t\right)-\left(\frac{H_{2}}{R}\right)^{2}}+\exp \left(-2 \tau \omega^{2} t\right) \sin ^{-1}\left(\frac{H_{2}}{R}\right)\right]
\end{aligned}
$$$$
\text { for } 1-\exp \left(-\tau \omega^{2} t\right) \leq \frac{H}{R} \leq 1+\exp \left(-\tau \omega^{2} t\right)
$$

$$
\frac{N}{N_{0}}=0 \quad \text { for } \quad \frac{H}{R} \geq 1+\exp \left(-\tau \omega^{2} t\right)
$$

where:

$$
\begin{aligned}
& y_{p}=\tau g t-\tau^{2} g\left(1-\exp ^{-t / \tau}\right) \\
& H=\frac{\tau g}{\omega} \sqrt{1-2 \cos (\omega t) \exp \left(-\tau \omega^{2} t\right)+\exp \left(-2 \tau \omega^{2} t\right)}
\end{aligned}
$$

for $\omega t<\pi$

$$
\begin{aligned}
& H=\frac{\tau g}{\omega}\left[1+\exp \left(-\tau \omega^{2} t\right)\right] \quad \text { for } \omega t>\pi \\
& H_{1}=\frac{R^{2}+H^{2}-R^{2} \exp \left(-2 \tau \omega^{2} t\right)}{2 H} \\
& H_{2}=H-H_{1}
\end{aligned}
$$

and $t$ here is the time begins as the first revolution begins. It should be noted that the parameter "time" in Moss's equation is different from that defined in Gruel's equation (or Eq. (1)).

The rotating drum has been extensively employed to study the retention of microorganisms in aerosol phase and the decay of bioaerosols under a variety of environmental conditions, such as relative humidity, temperature, UV, ozone, germicides, and gaseous pollutants (Krumins et al., 2008; Verreault et al., 2008; Piercy et al., 2010; Santarpia et al., 2012; Verreault et al., 2013; Santarpia et al., 2014; Verreault et al., 2014; Ratnesar-Shumate et al., 2015; Johnson et al., 2016; Turgeon et al., 2016; Haddrell and Thomas, 2017). However, the bioaerosol decay measured in these studies is a combination of biological decay and physical decay. It is important to evaluate the physical decay of aerosols in a rotating drum so as to elucidate the actual effectiveness of the studied parameters. Moreover, the deposition of aerosols is a function of particle size and it is crucial to characterize the physical decay for the bioaerosol size spectrum in a rotating chamber to optimize the rotation rate for prolonged aerosol suspension time period.

An endeavor has been made to experimentally investigate the aerosol physical decay as a function of particle size, suspension time, and rotation rate in a rotating chamber by Sutton and colleague (Embury and Sutton, 2005; Sutton, 2005). The aerosol decay experiments are conducted in a 1-m-diameter drum chamber with a piston inside the drum to pull air into or out of the drum which is different from Goldberg's rotating drum. However, some of the experimental results reveal a need for further improvements. First, the test particles failed to show a bimodal distribution with peaks at 3 and $6 \mu \mathrm{m}$ as expected due to triboelectric 
charging. The test particles were generated pneumatically in a large plastic bag using a sonic nozzle. The size distribution and concentration of challenged aerosols are not well controlled to minimize the measurement bias. Moreover, some experimental data are not realistic. For example, the aerosol decay results of 3-rpm test did not fall in line with those of 2- and 4-rpm tests and the concentrations of 1and $2-\mu \mathrm{m}$ particles increased within the first 4 to 6 hours for some of the tests. These discrepancies have not been addressed. Finally, Sutton's experimental measurements and Moss's model agreed only qualitatively but not quantitatively.

In this study, some improvements have been made to obtain reliable measurement data. The test aerosols were generated and neutralized before being introduced into the drum chamber to eliminate the measurement bias due to triboelectric charging. Furthermore, the initial aerosol concentration in the rotating drum was well controlled at a low concentration (approximately 170 particles $\mathrm{cm}^{-3}$ ) to reduce aerosol coagulation. The size distribution of the test aerosols meets the designated size range of different types of bioaerosols. To make the best use of rotating drum chambers in bioaerosol aging studies, this study aims to characterize the aerosol decay in a rotating drum, to determine the optimal rotation rate as a function of particle size, and to compare the theoretical predictions of Gruel's and Moss's models with experimental measurements.

\section{METHODS}

The experimental system comprises two compartments, the aerosol generation system and the rotatable drum unit as illustrated in Fig. 1. An ultrasonic atomizer (Model 8700120MS, Sono-Tek Corporation, Poughkeepsie, NY, USA) is adopted to generate micrometer-sized PST (potassium sodium tartrate tetrahydrate, density $1.79 \mathrm{~g} \mathrm{~cm}^{-3}$,
$\mathrm{KOCO}(\mathrm{CHOH})_{2} \mathrm{COONa} \cdot 4 \mathrm{H}_{2} \mathrm{O}$, A.C.S. grade, J.T.Baker ${ }$, Center Valley, PA, USA) particles as surrogates of bioaerosols. A syringe pump (KDS 200/200P, KD Scientific Inc., Holliston, MA, USA) is employed to deliver the solution to the ultrasonic atomizer to generate test particles. Then the particles are neutralized to the Boltzmann charge equilibrium using an aerosol neutralizer $\left(10-\mathrm{mCi}^{241} \mathrm{Am}\right.$ radioactive source). The aerosol output is diluted with filtered, dried compressed air in an aerosol capacitance chamber which is $20 \mathrm{~cm}$ in diameter and $100 \mathrm{~cm}$ in height. Previous research (Ho, 2006) showed that the aerosol decay can be biased by aerosol coagulation when the initial aerosol concentration exceeds $10^{5}$ particles $\mathrm{cm}^{-3}$. To avoid measurement bias due to aerosol coagulation, the dilution air is set at a flow rate of $60 \mathrm{~L} \mathrm{~min}^{-1}$. An aerodynamic particle sizer (APS; Model 3321, TSI Inc., St. Paul, MN, USA) is employed to measure the aerosol number concentration and distribution in the aerodynamic size range of $0.5-20 \mu \mathrm{m}$. The APS can measure particle number concentration up to 1000 counts $\mathrm{cm}^{-3}$ at diameters of 0.5 and $10 \mu \mathrm{m}$ with coincidence errors less than $5 \%$ and $10 \%$, respectively (TSI Inc., 2012).

The acrylic rotating drum is $0.29 \mathrm{~m}$ in inner diameter and $0.59 \mathrm{~m}$ in length with a total volume of $39 \mathrm{~L}$. The drum is mounted on a rack to allow free rotation on a horizontal axis and connected to an electric motor with a belt to attain the required rotation rate. Probes and sampling ports are installed on the non-rotating part of the drum. The temperature and relative humidity inside the chamber are monitored with a Temp/RH probe (HP 22, Rotronic Inc., Switzerland). A stainless tube, $0.34 \mathrm{~m}$ (length) $\times 4.2 \mathrm{~mm}$ (inner diameter), is inserted into the drum with the inlet of the sampling probe positioned in the center of the rotating chamber. The outlet of the sampling probe is directly connected to the inner nozzle of the APS. Before each
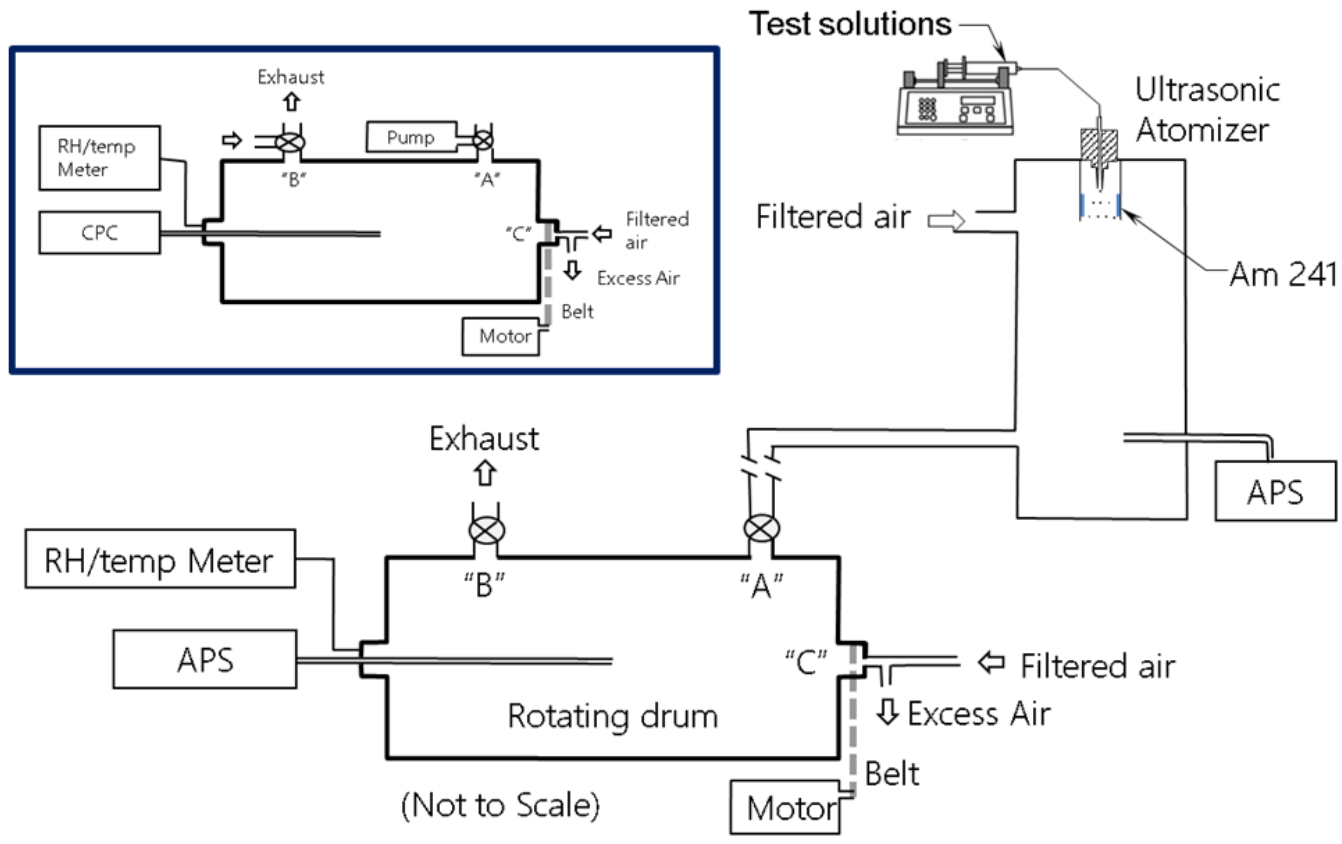

Fig. 1. Schematic diagram of the experimental system. 
experiment, the chamber is purged with filtered compressed air until no aerosols are detected by the APS. The test aerosols are introduced into the rotating drum through the " $A$ " valve and the excess air is vented through the " $B$ " valve as illustrated in Fig. 1. Aerosols are introduced into the drum chamber for about 6 minutes to reach a stable particle concentration of approximately 170 particles $\mathrm{cm}^{-3}$ during the drum experiments to prevent measurement bias due to coagulation effect. Then both " $\mathrm{A}$ " and "B" valves are closed and the air duct connecting the aerosol capacitance chamber and the rotating chamber is disconnected. The drum operates at the assigned rotating rate and the APS samples at $1 \mathrm{~L} \mathrm{~min}^{-1}$. To balance for air loss to sampling, $2 \mathrm{~L} \mathrm{~min}^{-1}$ filtered compressed air is supplied into the drum through "C" valve and the excess air is vented. All the measurements are conducted in triplicate unless otherwise stated.

To examine the aerosol decay over time, the mean aerosol concentration after certain suspension period is divided by the initial aerosol concentration to obtain the suspended aerosol fraction. Goldberg (1958) assumed that the aerosol decay in a rotating drum could be expressed as a constant percent loss per unit time by the following equation:

$$
N=N_{0} \exp ^{-k t}
$$

where $N_{0}$ is the initial aerosol number or concentration, $N$ is the aerosol number or concentration after time $t$, and $k$ is the aerosol decay constant. This first-order model for aerosol decay is governed by the particle aerodynamic properties and rotation rate of the drum.

\section{RESULTS AND DISCUSSION}

\section{Bias versus Sampling Protocols}

To evaluate the bias from dilution effect due to sampling, an experimental system is built as illustrated in the upper left box of Fig. 1. Ambient aerosols with a count median diameter (CMD) of $102 \mathrm{~nm}$ and a geometric standard deviation (GSD) of 1.98 are introduced into the rotating chamber to alleviate the effects of gravitational settling and centrifugal impaction. The total aerosol concentration is continuously monitored by a condensable particle counter (CPC; Model 3022A, TSI Inc., St. Paul, MN, USA) at an inlet flow rate of $1 \mathrm{~L} \mathrm{~min}^{-1}$ under the drum rotation rates of 0,5 , and $20 \mathrm{rpm}$, respectively. Fig. 2 shows the suspended aerosol fraction as a function of time during continuous sampling. No significant difference is found for the aerosol decay under different rotation rates. This is because the influence of gravity and rotation are both negligible for the sub-micrometer-sized aerosols being tested. As for aerosol dilution caused by sampling, the resulting decay constant is estimated to be $0.033 \mathrm{~min}^{-1}$. It can be inferred that the total sampling time for completing the experiment is not allowed to exceed 93 seconds assuming a 5\% tolerance of error. Otherwise, the dilution effect should be adjusted to obtain true decay constants.

According to the experimental setup shown in Fig. 1, it takes only 39 minutes to replace the air volume of the drum chamber under an ideal plug flow condition. Two sampling approaches including continuous sampling and intermittent sampling on the aerosol decay measurement are compared. The test aerosols with a CMD of $5.16 \mu \mathrm{m}$ and a GSD of 1.67 are generated and introduced into the rotating chamber. Owing to the small radius of the drum used in this work, the drum is operated at a rotation rate of $5 \mathrm{rpm}$ to retain more suspended particles than in a stationary drum chamber. For continuous sampling, the aerosol concentration in the rotating chamber is continuously monitored by the APS at $1 \mathrm{~L} \mathrm{~min}^{-1}$ throughout the $1-\mathrm{hr}$ suspension period. As for intermittent sampling, the APS samples for 1 minute only at the beginning and at the end of the 1-hr suspension period with no air sampling in between. Approximately 5\% air is withdrawn from the rotating chamber and the resulting bias on the aerosol decay measurement should be negligible. Fig. 3 compares

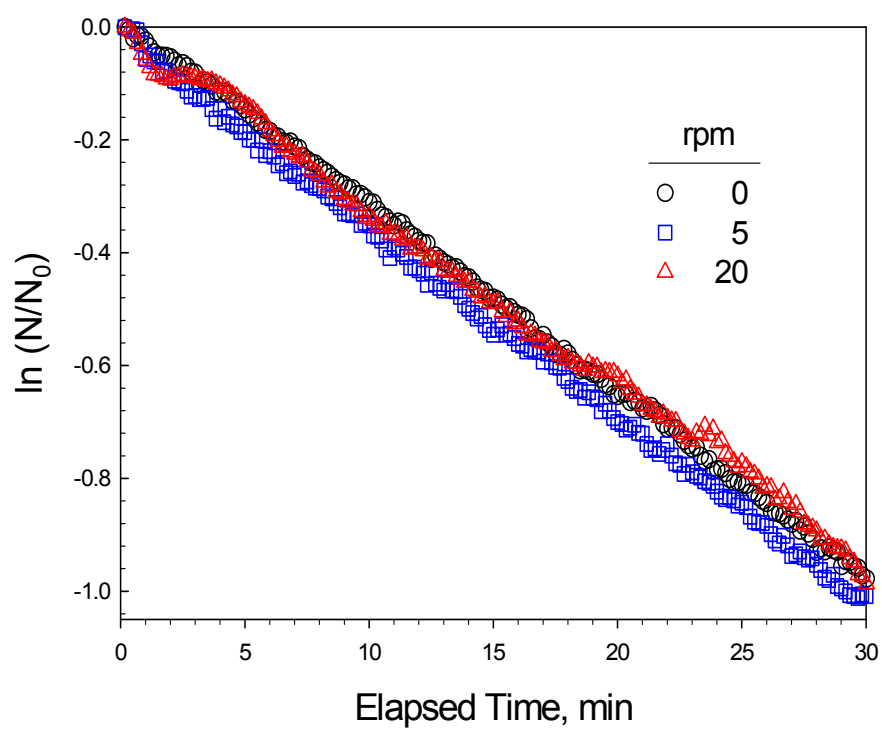

Fig. 2. Decay kinetics of ambient aerosols by continuous sampling under rotation rates of 0,5 , and $20 \mathrm{rpm}$. 
the suspended aerosol fraction versus particle size for both continuous and intermittent sampling after 1-hr suspension period. As is expected, larger particles decay faster than small particles due to gravitational settling. Moreover, the aerosol decay in the rotating chamber is significantly biased by continuous sampling and the calculated aerosol decay rates under continuous sampling are seven times more than those under intermittent sampling, especially for particles smaller than $5 \mu \mathrm{m}$. To avoid the bias from sampling protocol, the intermittent sampling approach with total sampling time not exceeding the abovementioned limitation (93 seconds) is adopted for the remaining experiments, unless otherwise specified.

\section{Aerosol Decay versus Particle Size}

The aerosol concentrations in the rotating drum with a rotation rate of $5 \mathrm{rpm}$ are measured by the APS for a sampling period of 15 seconds every 15 minutes throughout the 1-hr suspension period. Fig. 4 shows the aerosol decay over time for particle sizes of $1,3,5.1$, and $7.2 \mu \mathrm{m}$. The straight lines demonstrate that the aerosol concentrations for each particle size in the rotating chamber follow a firstorder exponential decay. Moreover, larger particles decay faster than smaller particles as expected. The calculated decay constants for 1-, 3-, 5.1-, and 7.2- $\mu \mathrm{m}$ particles are $0.0033 \pm 0.0006,0.0084 \pm 0.0003,0.016 \pm 0.002$, and 0.059 $\pm 0.005 \mathrm{~min}^{-1}$, respectively. These results imply that the aerosol decay kinetics inside the rotating drum for different particle sizes can be estimated using the extrapolation method according to the decay constant calculated using short-term measurements of aerosol decay kinetics.

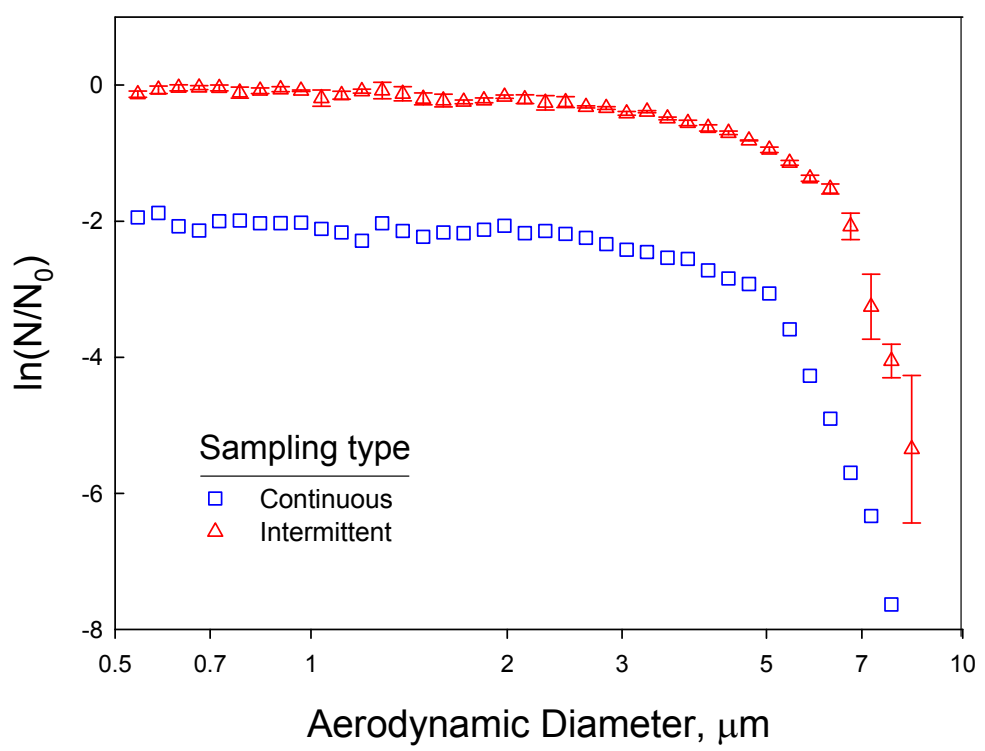

Fig. 3. Aerosol decay as a function of particle size for continuous and intermittent sampling for the duration of 1 hour at a rotation rate of $5 \mathrm{rpm}$.

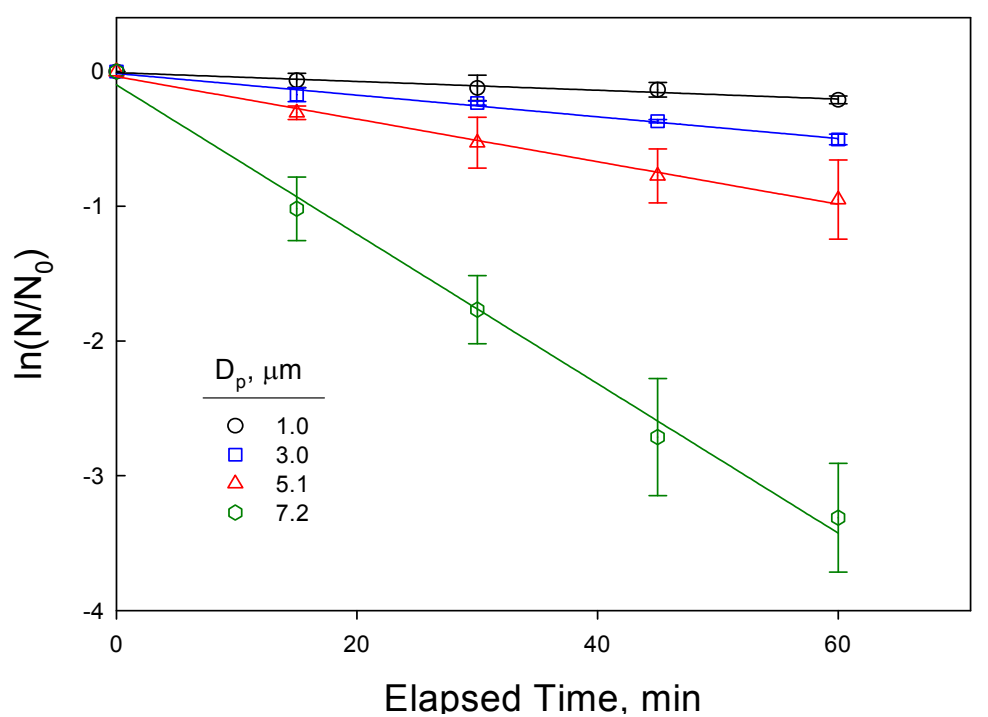

Fig. 4. Aerosol decay as a function of time for particles of different sizes. 


\section{Aerosol Decay as a Function of Time}

To evaluate the aerosol decay over time inside a rotating drum, the test aerosols with a CMD of $5.16 \mu \mathrm{m}$ and a GSD of 1.67 are generated and introduced into the drum which rotates at $5 \mathrm{rpm}$. The aerosol number concentrations as function of aerosol size, after rotating for 1,4 , and 24 hours are measured by the APS. Fig. 5 shows the fraction of initial particles that remain suspended as functions of particle size and suspension time. The aerosols in the rotating chamber decay dramatically with increasing suspension time. For $1-\mu \mathrm{m}$ particles, the suspended aerosol fractions are 92 , 73 , and $14 \%$ after 1,4 , and 24 hours of suspension, respectively. Moreover, aerosol decay tendency is more prominent for the beginning of the test as the particle size increases. For example, the suspended aerosol fraction for $2-\mu \mathrm{m}$ particles varies from $80 \%$ for $1-\mathrm{hr}$ suspension to $48 \%$ after 4 -hr suspension while the corresponding suspended aerosol fraction ranging from $92 \%$ to $73 \%$ for $1-\mu \mathrm{m}$ particles. The solid lines represent the results calculated using the extrapolation method according to the decay constants obtained from the aerosol decay kinetic measurements of 0.5 -hr suspension period. As can be observed, the calculated results match well with the measurements of 1, 4, and 24-hr suspension period. It is proved again that the aerosol concentration over an extended period of suspension time in a rotating chamber can be predicted using the decay constant measured within a limited time period.

Evidence shows that the retention of aerosols in rotating drums is less efficient than the theoretical predicted data (Frostling, 1973; Sutton, 2005; Verreault et al., 2013, 2014). To comprehensively compare the experimental measurements with the existing mathematical models, the fractions of suspended aerosols in the rotating drum as a function of particle size are calculated for the suspension durations of 1, 4, and 24 hours using Eqs. (1) and (3). Results of the

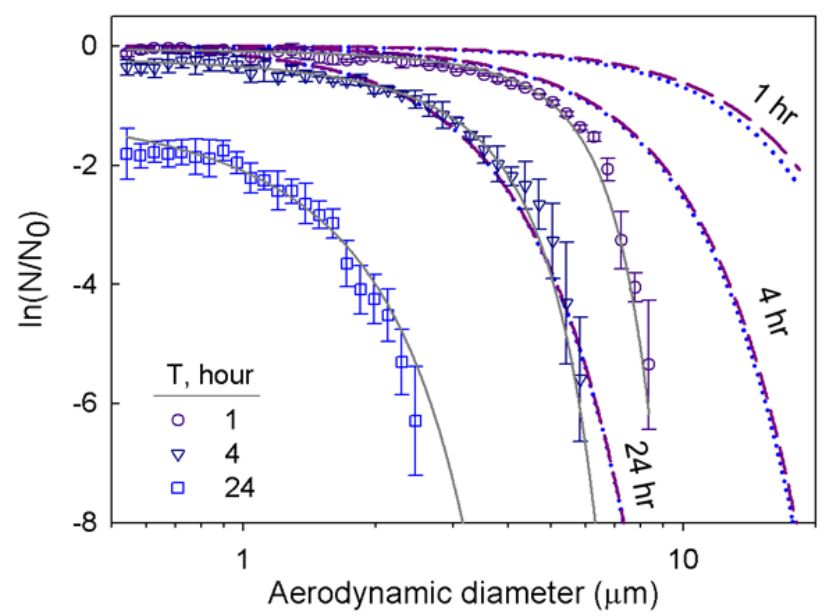

Fig. 5. Aerosol decay over time as a function of particle size at $5 \mathrm{rpm}$. Symbols represent experimental data and solid lines represent the suspended aerosol fraction calculated from the decay constant of a $0.5-\mathrm{hr}$ measurement period. Long-dashed lines and dotted lines represent the results of Gruel et al. (1987) and Asgharian and Moss (1992), respectively. calculations are also shown in Fig. 5 with the long-dashed and dotted lines representing the predictions of Gruel's model and Moss's model, respectively. Fig. 6 shows the ratio of experimental measurements and theoretical solutions using the two models. Both mathematical models are found to significantly underestimate the aerosol decay in the rotating drum. For 1-hr suspension time period, both Gruel's and Moss's models predict correctly the suspended aerosol fraction for particles smaller than $5 \mu \mathrm{m}$. For those particles larger than $5 \mu \mathrm{m}$, the differences between experimental and theoretical data may be partly attributed to measurement bias because there are fewer particles of larger size after suspension duration. In addition, as the challenged particles were injected into the drum chamber through the "A" valve, this will affect the flow pattern inside the drum and the path of the entering particles will inevitably affected. The aerosol concentration inside the chamber is initially not uniform. Eventually, the particles will become fairly uniform throughout the chamber volume as stirred settling model is appropriate for turbulent mixing due to the incoming particle flow and convective mixing due to small temperature differences between air and chamber wall (Fuchs, 1964). On the other hand, it should be noted that only gravitational and viscous drag forces are relevant and taken into consideration in the mathematical models of Gruel and Moss (Gruel et al., 1987; Asgharian and Moss, 1992). The aerosol motion along the horizontal axis is ignored. Forces due to electrostatics, diffusion, pressure gradient, and mutual collision are also not considered. In addition, as the drum is assumed to be maintained in an isothermal condition, thermal gradient forces are excluded in the models. In a real situation, the gravitational gyration becomes the dominant transport mechanism as the drum starts to rotate. Sutton (2005) claimed that electrostatic forces due to triboelectric charging play an important role in determining the aerosol decay. However, significant differences between our experimental data and Moss's mathematical model are still found after the effect of

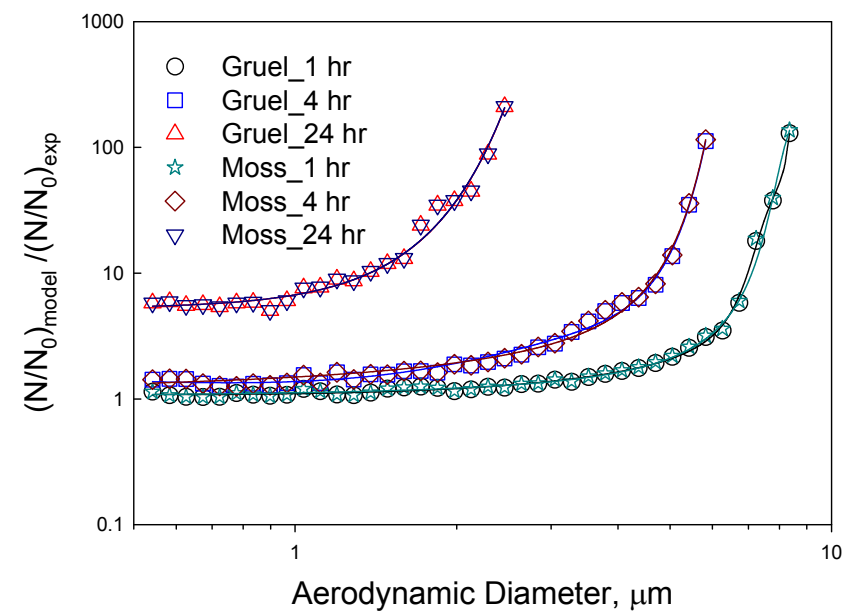

Fig. 6. Ratios of the experimental data and numerical data predicted by Gruel et al. (1987) and Asgharian and Moss (1992) as a function of particle size at $5 \mathrm{rpm}$ after 1-, 4-, and 24-hr suspension periods. 
electrostatic forces was excluded by aerosol neutralization in this work. Convective diffusion is another possible mechanism proposed by Sutton (2005). However, no satisfactory explanation can be made due to the limitation of this experiment.

\section{Aerosol Decay as a Function of Rotation Rate}

The fractions of suspended particles are measured at the rotation rate varying from 0 to $20 \mathrm{rpm}$ for 1-hr suspension time period. For better clarity, only the results of 0,5 , and $20 \mathrm{rpm}$ are plotted in Fig. 7. As can be seen, the rotating drum chamber yields more suspended particles at $5 \mathrm{rpm}$ than at stationary phase $(0 \mathrm{rpm})$ and $20 \mathrm{rpm}$. For $2-\mu \mathrm{m}$ particles, the fraction of suspended particles is $84 \%$ for $5 \mathrm{rpm}$ but only 14 and $45 \%$ for the drum rotated at 0 and $20 \mathrm{rpm}$, respectively. The experimental results imply that the rotation of a drum chamber greatly enhances particle suspension and the rotating rate can be optimized to retain the suspended particles for an extended time period.

The theoretical model predictions of Gruel and Moss for different rotation rates are also shown in Fig. 7 as longdashed and dotted lines, respectively. For the current drum geometry (drum radius $=14.5 \mathrm{~cm}$ ), particles larger than $1.5 \mu \mathrm{m}$ will deposit at the wall of a stationary drum $(0 \mathrm{rpm})$ for 1-hr measurement time as estimated by Eq. (2) (also referred to as Moss's model). The mathematical model underestimated the fraction of suspended particles in a stationary drum. The underestimation may be due to convective or turbulent mixing caused by air compensation for sampling loss. When the drum starts to rotate, both models predict identical results on the fraction of suspended particles at high rotation rate $(20 \mathrm{rpm})$ and yield slight differences at low rotation rate $(5 \mathrm{rpm})$ indicating the combined effect of gravitational settling and centrifugal deposition. In general, mathematical models tend to overestimate the fraction of suspended particles when the drum chamber is rotating and this is in good agreement with the findings of previous

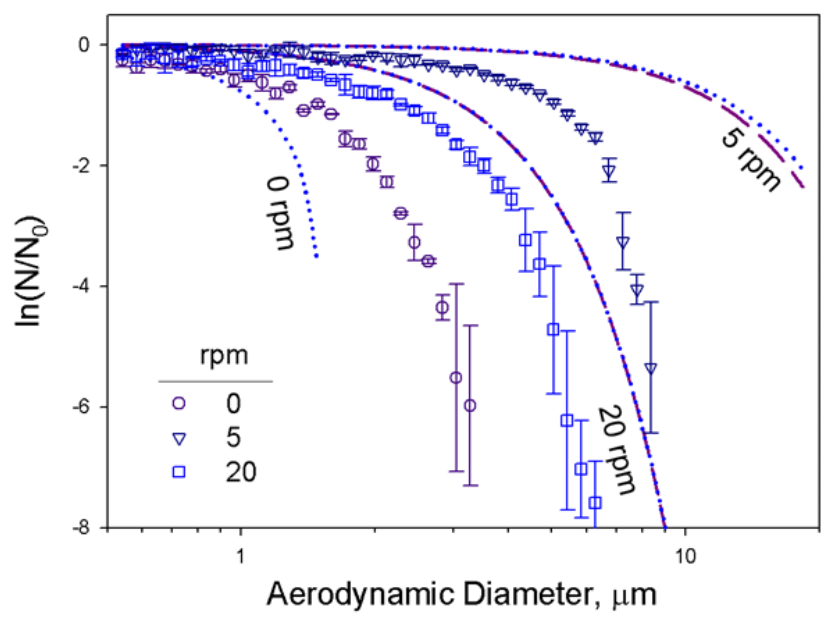

Fig. 7. Aerosol decay as a function of particle size. Symbols represent experimental data under different rotation conditions. Long-dashed lines and dotted lines represent the results of Gruel et al. (1987) and Asgharian and Moss (1992), respectively. studies (Embury and Sutton, 2005; Sutton, 2005). Fig. 8 shows the ratio of experimental measurements and numerical data at 0,5 , and $20 \mathrm{rpm}$, as a function of particle size. Both mathematical models predict accurately the suspended aerosol fraction for particles smaller than $5 \mu \mathrm{m}$ at $5 \mathrm{rpm}$. However, particle residence is dramatically overestimated especially for larger particles at higher rotation rate. As mentioned above, the real case is likely very complicated whereas only gravitational and viscous drag forces were taken into consideration in the existing models.

\section{Optimal Rotation Rate versus Particle Size}

The particle size and rotation rate-dependent aerosol decay constants are plotted in Fig. 9. It is evident that there is a minimal decay constant for each particle size in the measured rotation rate range, implying that the drum rotation rate can be optimized to retain suspended particles for an extended time period during the experiments when the gravity force and centrifugal force are comparable in magnitude. An interesting trend of increase in optimal rotation rate with increasing particle size is observed. The optimal rotation rate as a function of particle size is shown in Fig. 10 and the optimal rotation rate (V in rpm) is found to be accurately predicted with particle size $\left(d_{p}\right.$ in $\left.\mu \mathrm{m}\right)$ by the following equation:

$\mathrm{V}=0.6007 d_{p}+1.9159$

The optimal rotation rate varies from 2 to $7 \mathrm{rpm}$ in the particle size range of 1 to $7 \mu \mathrm{m}$ and is proportional to particle size. This finding is in agreement with Sutton's experimental results that the optimal rotation rate falls between 1 and $6 \mathrm{rpm}$ (Sutton, 2005). As a rough estimate, it would be a good guess to choose $5 \mathrm{rpm}$ for prolonged residence time periods for the potential bioaerosol size spectrum in aging studies based on our experimental findings. However, such predicted result is inconsistent with the numerical studies of Gruel et al. (1987) and Asgharian and

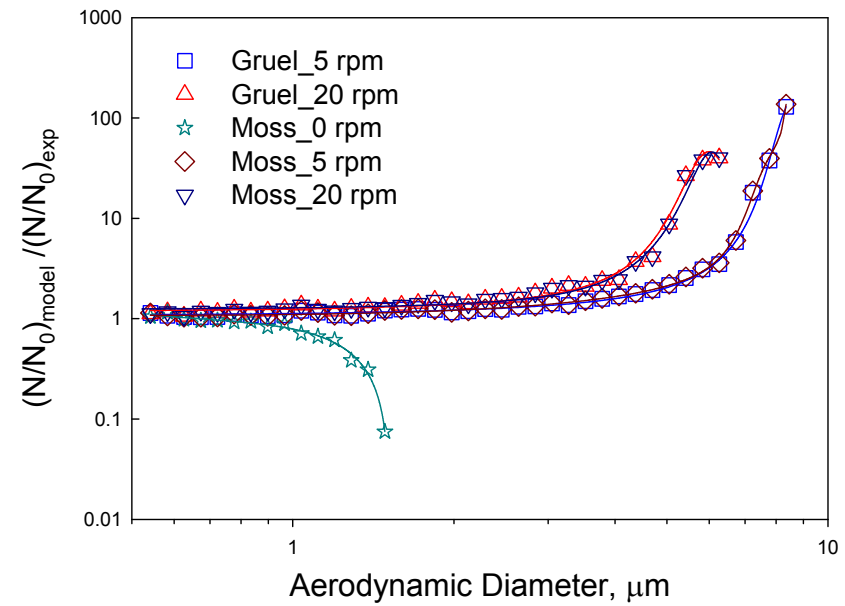

Fig. 8. Ratios of experimental data and numerical data respectively predicted by Gruel et al. (1987) and Asgharian and Moss (1992) as a function of particle size at 0,5 , and $20 \mathrm{rpm}$ after 1-hr suspension period. 


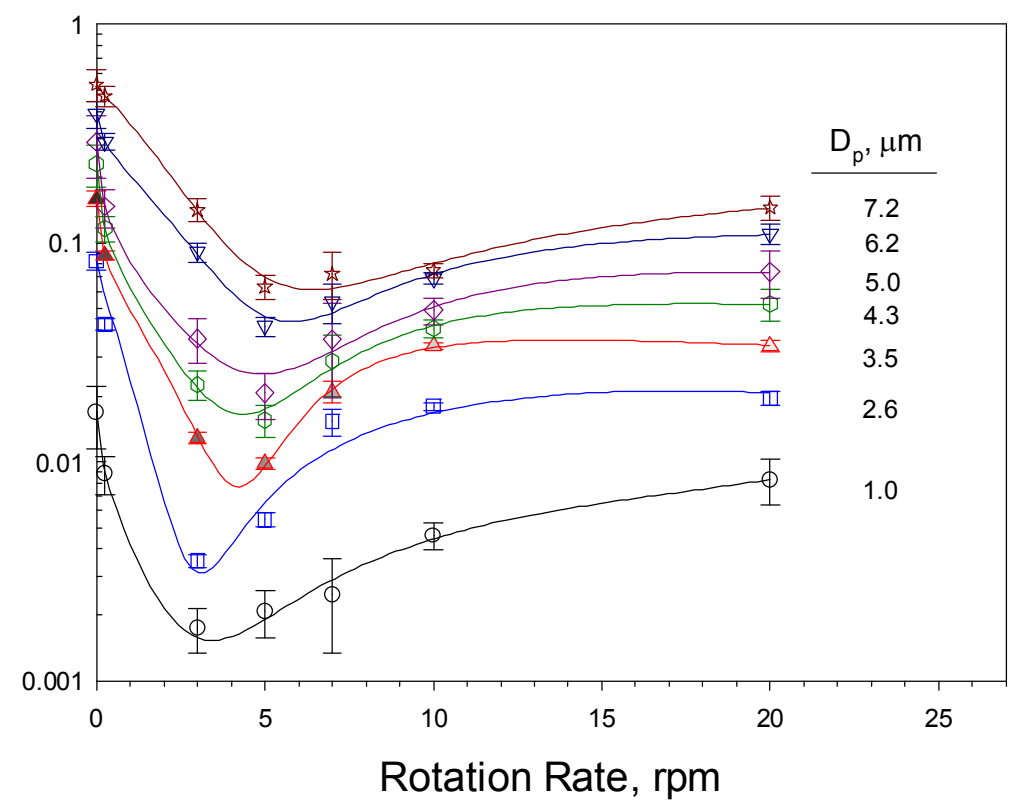

Fig. 9. Effect of rotation rate on aerosol decay constant as a function of particle size.

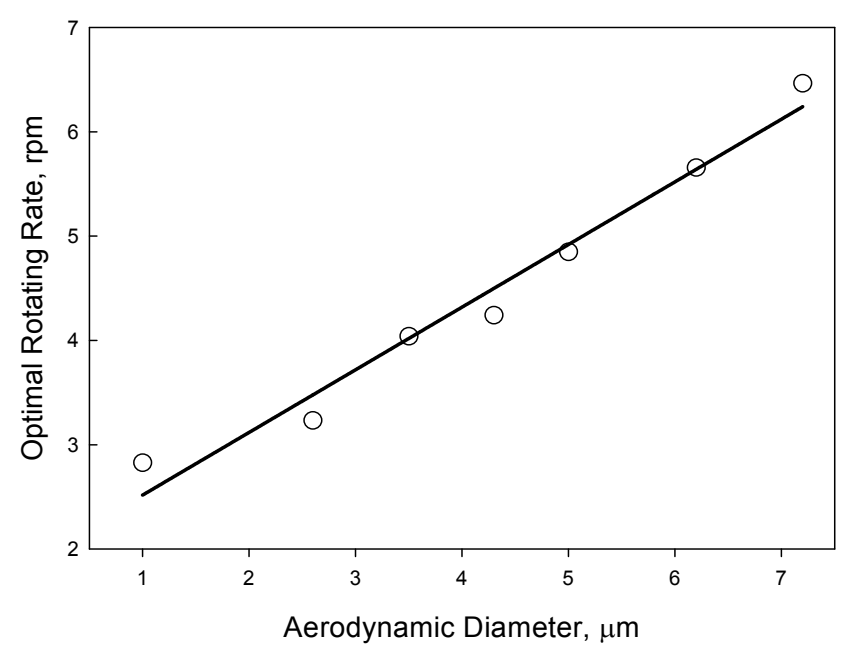

Fig. 10. Optimal rotation rates versus particle size.

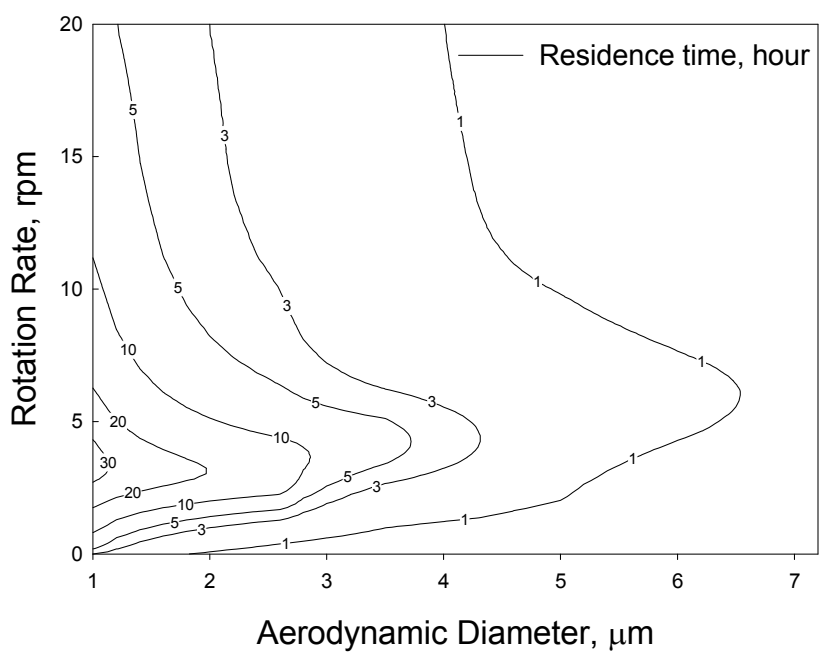

Fig. 11. Aerosol retention time isopleths.
Moss (1992). The optimal rotation rate is concluded to be independent of particle size for particles smaller than $10 \mu \mathrm{m}$ (Gruel et al., 1987) or $5 \mu \mathrm{m}$ (Asgharian and Moss, 1992). Moreover, the optimal rotation rates predicted by Moss's model for all particle sizes are quite similar and vary from 0.29 to $1.41 \mathrm{rpm}$ which is much lower than the experimental results obtained in this study and the previous study (Embury and Sutton, 2005).

For practical application of a rotating drum chamber in bioaerosol aging experiments, it is critical to retain the test microorganisms in aerosol phase throughout the experimental process. Fig. 11 shows aerosol residence time isopleths. Aerosol residence time is defined as the time that $5 \%$ of initial suspended particles remain suspended and is calculated given the rotation rate and aerosol decay constant are known. With the current drum geometry used in this work, the optimal rotation rate between 2 and $4 \mathrm{rpm}$ can suspend $1-\mu \mathrm{m}$ particles for over 24 hours. The operating range of drum rotation rate for large particles and long residence time is narrower than that for small particles and short residence time, implying greater care is required when adjusting the optimal rate for large particles and long suspension duration.

\section{CONCLUSIONS AND RECOMMENDATIONS}

This study investigates the effects of various critical parameters on the decay constants of aerosols in a drum chamber. The experimental results are also compared with theoretical predictions from two previous models of aerosol dynamics in a rotating drum. Since the continuous sampling approach underestimates the aerosol decay constants due to the dilution effect, the following conclusions are drawn from experimental results based on the intermittent sampling protocol.

For micrometer-sized particles, a considerable delay of sedimentation can be achieved using a rotating drum. The 
evolution of the aerosol concentration in a rotating chamber over an extended period can be predicted using the decay constant which is measured within a limited time period. With the current drum geometry, the optimal rotation rate varies from 2 to $7 \mathrm{rpm}$ for the particle size range of 1 to 7 $\mu \mathrm{m}$ and is linearly proportional to the particle size. At the optimal rotation rate $(2-4 \mathrm{rpm})$ for $1-\mu \mathrm{m}$ particles, $5 \%$ of them can remain suspended for over 24 hours. This rate, however, must be adjusted for large particles in order to maximize the period of suspension. Although unresolved discrepancies remain between Moss's model and the experimental data, the rotating drum chamber is still a useful tool, and a rate of $5 \mathrm{rpm}$ is recommended for bioaerosol aging studies.

\section{ACKNOWLEDGMENTS}

This research was financially supported by the Ministry of Science and Technology, Taiwan, R.O.C., under the grant: MOST 103-2221-E-273-002-MY2.

\section{REFERENCES}

Asgharian, B. and Moss, O.R. (1992). Particle suspension in a rotating drum chamber when the influence of gravity and rotation are both significant. Aerosol Sci. Technol. 7: 263-277.

Chen, J.W., Lee, W.M., Chen, K.J. and Yang, S.H. (2016). Control of bioaerosols in indoor environment by filter coated with nanosilicate platelet supported silver nanohybrid (AgNPs/NSP). Aerosol Air Qual. Res. 16: 2198-2207.

Embury, J.F. and Sutton, T.A. (2005). Analysis of aerosol aging in the rotating drum chamber. No. ECBC-TR455. Edgewood Chemical Biological Center Aberdeen Proving Ground MD, USA.

Frostling, H. (1973). A rotating drum for the study of toxic substances in aerosol form. J. Aerosol Sci.4: 411-419.

Fuchs, N.A. (1964). The mechanics of aerosols. Macmillan Company, New York, USA.

Goldberg, L.J. (1971). Naval biomedical research laboratory, programmed environment, aerosol facility. Appl. Microbiol. 21: 244-252.

Goldberg, L.J., Watkins, H.M.S., Boerke, E.E. and Chatigny, M.A. (1958). The use of a rotating drum for the study of aerosols over extended periods of time. Am. J. Epidemiol. 68: 85-93.

Gruel, R.L., Reid, C.R. and Allemann, R.T. (1987). The Optimum Rate of Drum Rotation for Aerosol Aging. $J$. Aerosol Sci. 18: 17-22.

Haddrell, A.E. and Thomas, R.J. (2017). Aerobiology: Experimental considerations, observations, and future tools. Appl. Environ. Microbiol. 83: 00809-00817.

Ho, K.T. (2006). Evaluation of test method of indoor air cleaners. Master thesis. Institute of Occupational Medicine and Industrial Hygiene, College of Public Health, National Taiwan University, Taiwan.

Huang, H.L., Lee, M.G. and Tai, J.H. (2012). Controlling indoor bioaerosols using a hybrid system of ozone and catalysts. Aerosol Air Qual. Res. 12: 73-82.

Johnson, G.R., Knibbs, L.D., Kidd, T.J., Wainwright, C.E., Wood, M.E., Ramsay, K.A., Bell, S.C. and Morawska, L. (2016). A novel method and its application to measuring pathogen decay in bioaerosols from patients with respiratory disease. PLoS One 11: e0158763.

Krumins, V., Son, E. K., Mainelis, G. and Fennell, D.E. (2008). Retention of inactivated bioaerosols and ethene in a rotating bioreactor constructed for bioaerosol activity studies. CLEAN - Soil, Air, Water 36: 593-600.

Kuo, Y.M. (2015) Field evaluation of sampling bias with plastic petri dishes for size-fractionated bioaerosol sampling. Aerosol Sci. Technol. 49: 127-133.

Pich, J. (1972). Theory of gravitational deposition of particles from laminar flows in channels. J. Aerosol Sci. 3: 351-361.

Piercy, T.J., Smither, S.J., Steward, J.A., Eastaugh, L. and Lever, M.S. (2010). The survival of filoviruses in liquids, on solid substrates and in a dynamic aerosol. $J$. Appl. Microbiol. 109: 1531-1539.

Ratnesar-Shumate, S., Pan, Y. L., Hill, S.C., Kinahan, S., Corson, E., Eshbaugh, J. and Santarpia, J.L. (2015). Fluorescence spectra and biological activity of aerosolized bacillus spores and MS2 bacteriophage exposed to ozone at different relative humidities in a rotating drum. J. Quant. Spectrosc. Radiat. Transfer 153: 13-28.

Santarpia, J.L., Pan, Y. L., Hill, S.C., Baker, N., Cottrell, B., McKee, L., Ratnesar-Shumate, S. and Pinnick, R.G. (2012). Changes in fluorescence spectra of bioaerosols exposed to ozone in a laboratory reaction chamber to simulate atmospheric aging. Opt. Express 20: 2986729881.

Santarpia, J.L., Sanchez, A.L., Lucero, G., Servantes, B. and Hubbard, J. (2014). A laboratory exposure system to study the effects of aging on super-micron aerosol particles. Sandia national laboratories. No. SAND2014-0997, Sandia National Laboratories, Albuquerque, NM, USA.

Sutton, T. (2005). Decay of particle concentration as a function of rotation rate in a rotating drum chamber. No. ECBC-TR-436, Edgewood Chemical Biological Center Aberdeen Proving Ground MD, USA.

TSI Inc. (2012). Model 3321 aerodynamic particle sizer spectrometer operation and service manual, $\mathrm{P} / \mathrm{N}$ 1930092, Revision G. St. Paul, MN: TSI, Inc., USA.

Turgeon, N., Michel, K., Ha, T. L., Robine, E., Moineau, S. and Duchaine, C. (2016). Resistance of aerosolized bacterial viruses to four germicidal products. PLoS One 11: e0168815.

Verreault, D., Duchaine, C., Marcoux-Voiselle, M., Turgeon, N. and Roy, C.J. (2014). Design of an environmentally controlled rotating chamber for bioaerosol aging studies. Inhalation Toxicol. 26: 554-558.

Verreault, D., Killeen, S.Z., Redmann, R.K. and Roy, C.J. (2013). Susceptibility of monkeypox virus aerosol suspensions in a rotating chamber. J. Virol. Methods 187: 333-337.

Verreault, D., Moineau, S. and Duchaine, C. (2008). Methods for sampling of airborne viruses. Microbiol. Mol. Biol. Rev. 72: 413-444. 
Wingstedt, E.M.M., Pedersen, K. and Reif, B.A.P. (2009).

A modelling study of the flow pattern in a rotating drum for aerosol aging. Norwegian Defense Research Establishment (FFI) report 2009/01079.
Received for review, May 14, 2018 Revised, October 14, 2018 Accepted, October 21, 2018 